\title{
PROGRESS TOWARD THE STUDY OF LABORATORY SCALE, ASTROPHYSICALLY RELEVANT, TURBULENT PLASMAS
}

\author{
C.C. KURANZ ${ }^{1}$, R.P. DRAKE ${ }^{1}$, D.R. LEIBRANDT ${ }^{1}$, E.C. HARDING ${ }^{1}$, H.F. ROBEY ${ }^{2}$, \\ A.R. MILES ${ }^{2}$, B.E. BLUE ${ }^{2}$, J.F. HANSEN ${ }^{2}$, H. LOUIS ${ }^{2}$, M. BONO ${ }^{2}$, J. KNAUER ${ }^{3}$, \\ D. ARNETT ${ }^{4}$ and C.A. MEAKIN ${ }^{4}$ \\ ${ }^{1}$ University of Michigan \\ ${ }^{2}$ Lawrence Livermore National Laboratory \\ ${ }^{3}$ Laboratory for Laser Energetics \\ ${ }^{4}$ University of Arizona
}

(Accepted July 2004)

\begin{abstract}
Recent results from an ongoing series of Rayleigh-Taylor instability experiments being conducted on the Omega Laser are described. The goal of these experiments is to study, in a controlled laboratory setting, the mixing that occurs at an unstable interface subjected to an acceleration history similar to the explosion phase of a core-collapse supernova. In a supernova, the Reynolds number characterizing this mixing is extremely large $\left(\operatorname{Re}>10^{10}\right)$ and is more than sufficient to produce a turbulent flow at the interface. In the laboratory experiment, by contrast, the spatial scales are much smaller, but are still sufficiently large $\left(\operatorname{Re}>10^{5}\right)$ to support a turbulent flow and therefore recreate the conditions relevant to the supernova problem. The data from these experiments will be used to validate astrophysical codes as well as to better understand the transition to turbulence in such high energy density systems. The experimental results to date using two-dimensional initial perturbations demonstrate a clear visual transition from a well-ordered perturbation structure consisting of only a few modes to one with considerable modal content. Analysis of these results, however, indicates that while a turbulent spectrum visually appears to be forming, the layer has not yet reached the asymptotic growth rate characteristic of a fully turbulent layer. Recent advances in both target fabrication and diagnostic techniques are discussed as well. These advances will allow for the study of well-controlled $3 \mathrm{D}$ perturbations, increasing our ability to recreate the conditions occurring in the supernova.
\end{abstract}

\section{Introduction}

Laboratory astrophysics provides a link between astrophysical observations and models. These hydrodynamics experiments support the effort to model astrophysical systems. They are also compared to observations made in astrophysics. Intense lasers can create large energy densities in targets of mm-scale volume. These targets are well scaled to a supernova explosion so that the two will have similar hydrodynamic evolution (Ryutov et al., 1999).

The rapid collapse of the Fe core of a supernova causes a shockwave to move outward through the star. When the dense core is accelerated by a blast wave into the less dense outer layers, the Richtmeyer-Meshchov (Richtmyer, 1960; Meshkov, 1969) instability and the Rayleigh-Taylor (Rayleigh, 1900; Taylor, 1950) instability

Astrophysics and Space Science 298: 9-16, 2005.

(C) Springer 2005 


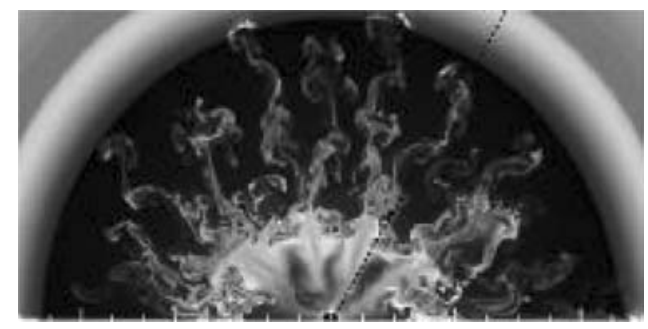

Figure 1. Simulation of supernova explosion.

occur. Structures evolve as the dense layer is decelerated by the less dense outer layer. Simulations of a supernova show spikes of heavier elements move outward into the less dense hydrogen layer, while bubbles of hydrogen penetrate inward. Kifondis et al. (2000) simulated the explosion with perturbations produced by neutrino-driven convection but did not include a perturbation at the outer surface. It is shown in Figure 1. These simulations under-predict the observed radial extent of material mixing in supernova. In SNe, the Rayleigh-Taylor instability generates outwardly propagating spikes of Fe group elements. The radial velocity of these spikes in simulations is lower than the observed value by a factor of approximately 2. In part the spikes are slowed when they encounter the dense material at the $\mathrm{He} / \mathrm{H}$ interface (Kifonidis et al., 2000). Fryxell, Müller, and Arnett had another 2D model that not include neutrino-driven convection, but did have a random perturbation on the entire volume. There was penetration of heavy elements, but not at velocities high enough to explain observations (Fryxell et al., 1991).

Laboratory experiments are being used to investigate two possible explanations for this mixing discrepancy. The Reynolds number characterizing mixing in supernova explosions is estimated to be of order $10^{10}$ (Ryutov et al., 1999). By comparison with classical flows, this should result in turbulent flow. None of the simulations done to date, however, appear to be turbulent. A transition to turbulence will provide an increase in the radial transport of material. Also, the initial conditions in the progenitor might give rise to an asymmetrical explosion, which would again result in enhanced radial transport of a fraction of the core material. Both numerical simulations and observations suggest the possibility of asymmetrical explosions. Laboratory experiments can be valuable in providing data on the transition to turbulence in similar instability-driven, high-Reynolds-number systems.

\section{2D, 2-Mode Coupling Target Design and Experiment}

The experiments are conducted in mm-scale shock-tubes that create similar interfacial acceleration history to that of supernova. This acceleration history can be seen in Figure 2. Figure 3 shows the target design for the experiment. The target is 
Interface trajectory

Omega expt. time scale (ns)

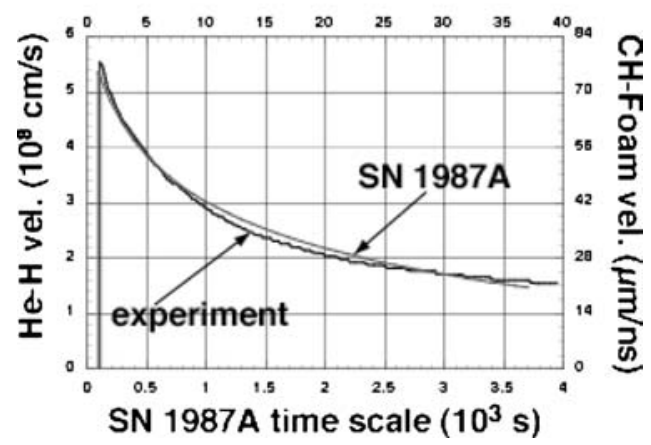

Figure 2. Acceleration history of SN1987A and experiment.

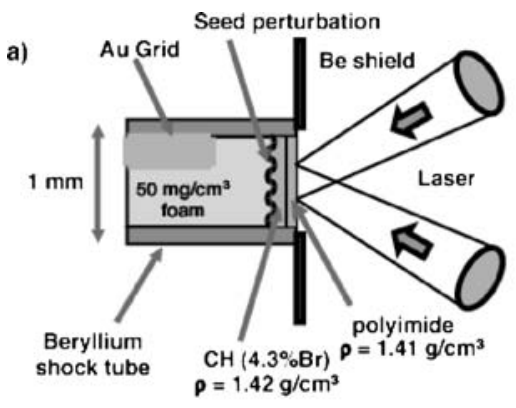

b)

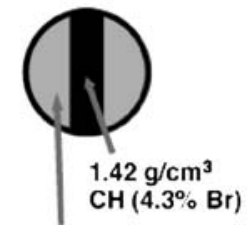

$1.41 \mathrm{~g} / \mathrm{cm}^{3}$ polyimide

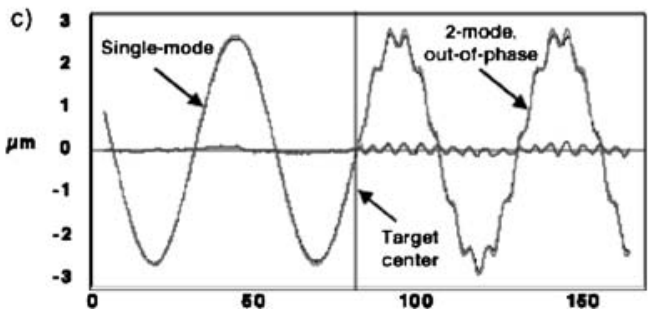

Figure 3. (a) Target schematic. (b) A face-on view of the rear surface of the polyimide with the doped $\mathrm{CH}$ strip in place. (c) Plot of single-mode perturbation and 2-mode perturbation with out of phase variation.

$800 \mu \mathrm{m}$ in diameter with a front surface of polyimide. Ten Omega (Boehly et al., 1995) laser beams with a 1 ns flat-topped pulse irradiate the polyimide surface with an intensity of approximately $10^{15} \mathrm{~W} / \mathrm{cm}^{2}$. The laser has a wavelength of $0.35 \mu \mathrm{m}$ and a spot size of $820 \mu \mathrm{m}$ FWHM. The ablated polyimide surface is $150 \mu \mathrm{m}$ thick with a density of $1.41 \mathrm{~g} / \mathrm{cm}^{3}$. The rear surface of the polyimide has a $200 \mu \mathrm{m}, 75 \mu \mathrm{m}$ deep $\mu \mathrm{m}$ opening and a strip of material glued into it that is $\mathrm{CH}$ doped with 4.3 at.\% of bromine or 3 at.\% iodine. A face-on view of the rear surface of the polyimide is 
shown in Figure 3b. The strip is density matched to the surrounding material and is also low $\mathrm{Z}$ so that the two materials will have similar hydrodynamic responses to extreme pressure. $\mathrm{X}$-rays are used to make a radiograph during the experiment. Since doped CH strongly absorbs the X-rays one can better observe the structure formed during the experiment. A 2 dimensional perturbation is machined onto the rear surface of the polyimide/doped $\mathrm{CH}$ package. The amplitude of this perturbation is $a_{0} \sin \left(k_{x} x\right)$, where $a_{0}=2.5 \mu \mathrm{m}$ and the wave number $k_{x}=2 \pi /(50 \mu \mathrm{m})$. On top of the initial perturbation this experiment included a second perturbation with a variation in phase. The target was split between a single mode perturbation and a 2 -mode perturbation. A plot of this amplitude can be seen in Figure 3c. Behind the polyimide/doped $\mathrm{CH}$ package is approximately $2.4 \mathrm{~mm}$ thick carbonized resorcinol formaldehyde $(\mathrm{CrF})$ foam. The foam has a density of $50 \mathrm{mg} / \mathrm{cm}^{3}$. This package is held together by a Be shock tube $1100 \mu \mathrm{m}$ in outer diameter and the front end of the target has a $2.5 \mathrm{~mm}$ Be shield to prevent interference with the diagnostics. A gold grid is placed on the target facing the diagnostic for location and magnification calibration.

The primary diagnostic in these experiments is X-ray radiography. These experiments used a point projection backlighting technique to increase resolution and contrast. A Ti foil was irradiated with several Omega laser beams, creating X-rays. The X-rays were focused through a $10 \mu \mathrm{m}$ pinhole in Ta through the target and to a single-strip framing camera.

\section{2D, 2-Mode Simulations}

The experimental setup for the 2-dimensional mode coupling experiment was simulated using the CALE code. CALE is a 2D Arbitrary Eulerian Lagrangian radiation hydrodynamics code. These simulations can be seen in Figures $4 \mathrm{a}-4 \mathrm{~d}$. Each figure is at a different time in the simulations and compares the single mode, 2-mode in-phase and 2-mode out-of-phase. The simulations are at $7 \mathrm{~ns}, 16 \mathrm{~ns}, 26 \mathrm{~ns}$, and $38 \mathrm{~ns}$. The simulations of this experiment show clear difference resulting from the initial phase of the two modes. The out-of-phase initial condition clearly produces a mixed layer of greater complexity.

\section{Experimental Results for 2D, 2-Mode Coupling Experiment}

Figures $5 \mathrm{a}-5 \mathrm{c}$ are the radiographs from the 2D mode coupling experiment at times of $13 \mathrm{~ns}, 25 \mathrm{~ns}$, and $37 \mathrm{~ns}$ respectively. These radiographs show a clear transition toward a more complicated modal structure, with clear difference from the supernova simulation as seen in Figure 1. By taking a horizontal lineout measurement of the spike and bubble one is able to calculate the amplitude of the mixing layer. Figure 6 is a plot of the average amplitude of the mixed layer vs. time. The solid lines are predictions from simulations and the different plot values represent single-mode, 

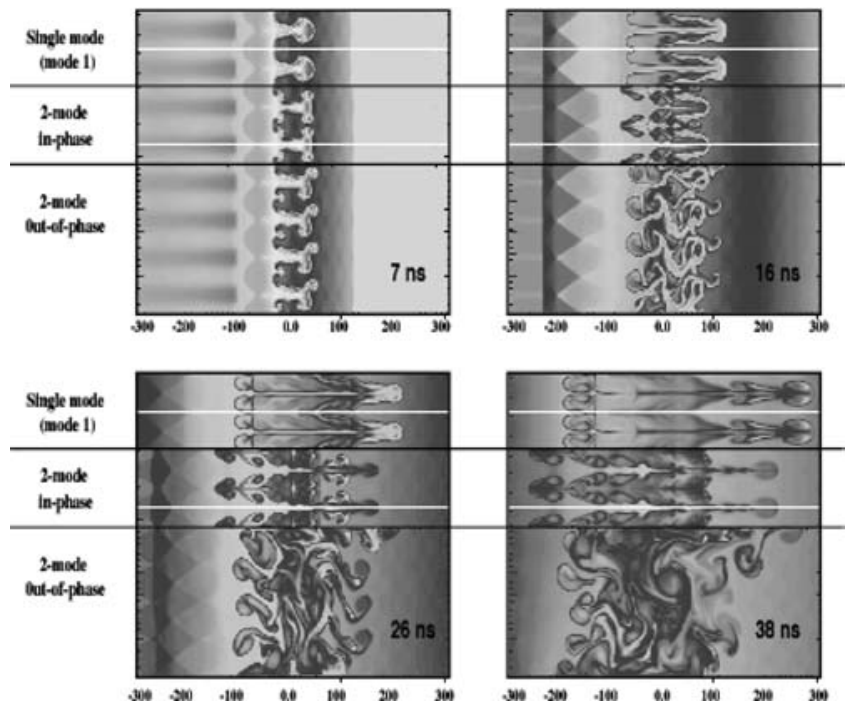

Figure 4. 2D CALE simulations single mode, 2-mode out-of-phase, and 2-mode in-phase perturbations at $t=7 \mathrm{~ns}, t=16 \mathrm{~ns}, t=25 \mathrm{~ns}, t=38 \mathrm{~ns}$.
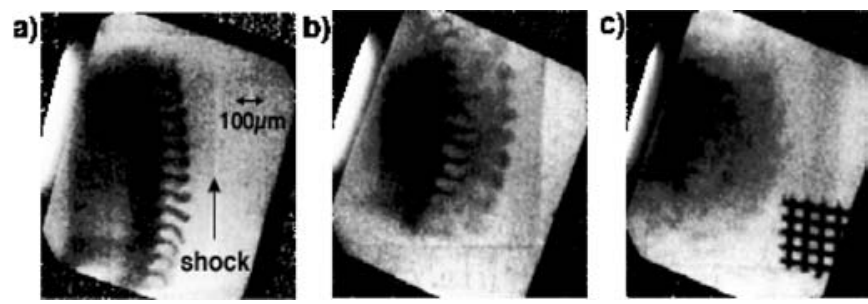

Figure 5. X-ray radiograph of 2D mode coupling experiment, (a) $t=13 \mathrm{~ns}$, (b) $t=25 \mathrm{~ns}$, (c) $t=38 \mathrm{~ns}$.

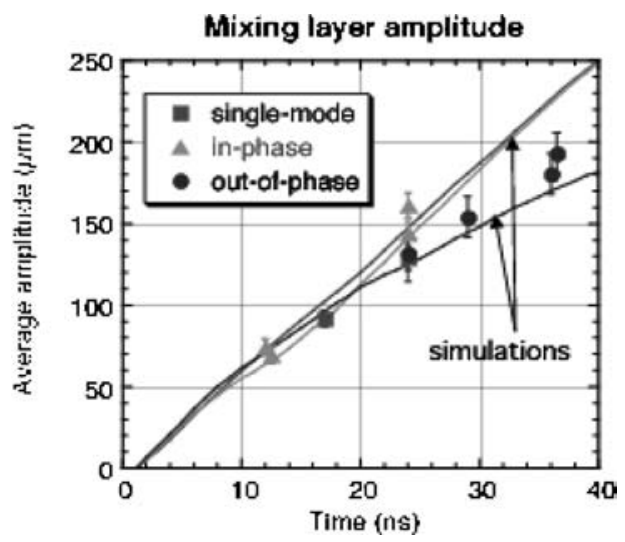

Figure 6. Plot of amplitude vs. time for 2D mode coupling experiment for single-mode, in-phase, and out-of-phase. 


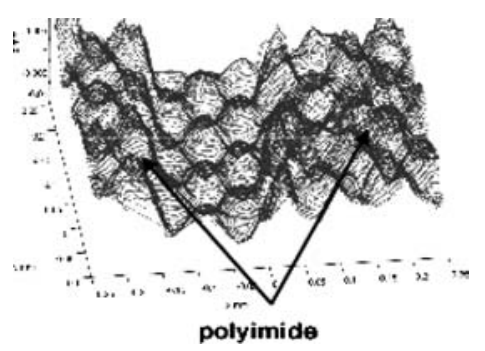

Figure 7. Mean elevation of the doped $\mathrm{CH}$ tracer strip is lower by $5 \mu \mathrm{m}$ compared to polyimide.

2-mode in-phase, and 2-mode out-of-phase experiments. This plot shows that even though the interface is increasing in modal complexity there is no increased in the overall layer growth rate has been observed. Some experimental issues limit the observational time, such as the edge effects from the shock tube walls. Also, the blast-wave strength decays with time, which impedes late-time growth. The upcoming National Ignition Facility will eventually allow for greater length scales and much longer evolution times.

\section{3D Perturbation Experiments}

Experiments were also performed with a 3D surface perturbation. An "egg-crate" perturbation was machined onto the rear surface of the polyimide/doped $\mathrm{CH}$ package. The amplitude of this perturbation is $a_{0} \sin \left(k_{x} x\right) \sin \left(k_{y} y\right)$, where $a_{0}=2.5 \mu \mathrm{m}$ and the wave numbers $k_{x}=k_{y}=2 \pi /(71 \mu \mathrm{m})$. These experiments have resulted in significantly increased spike growth. After the experiment was performed it was realized that the method used to machine the polyimide/doped $\mathrm{CH}$ surface introduced short-scale roughness in the doped $\mathrm{CH}$ strip. In addition, the interface had an unintentional large-scale (low-mode number) 2D perturbation that can be seen in the mean elevation of the tracer strip in Figure 7. Therefore, the initial surface perturbation has both shorter and longer components than the fundamental 3D mode.

The 3D experiments used an area backlighter as opposed to a pinhole backlighter that was used in the 2D mode coupling experiment. A thin Sc foil was placed approximately $4 \mathrm{~mm}$ from the target and irradiated on either side with additional laser beams. These beams were delayed 10 to $30 \mathrm{~ns}$ relative to the drive beams to monitor the evolution of the interface over time. The result is several radiographs at different times.

\section{3D Experimental Results}

$\mathrm{X}$-ray radiographs of the 3D experiments can be seen in Figure 8. The top row of radiographs used a $3 \mathrm{D}$ perturbation and the second row had a planar interface used 

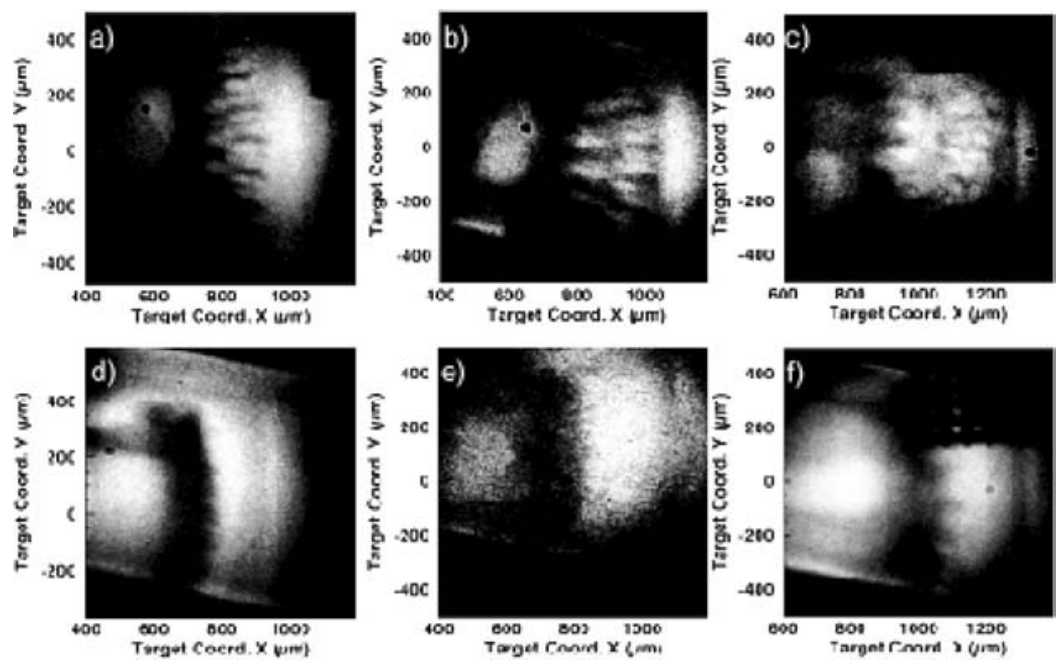

Figure 8. X-ray radiographic data for a three-dimensional perturbed interface and a planar interface in the top and bottom row, respectively.
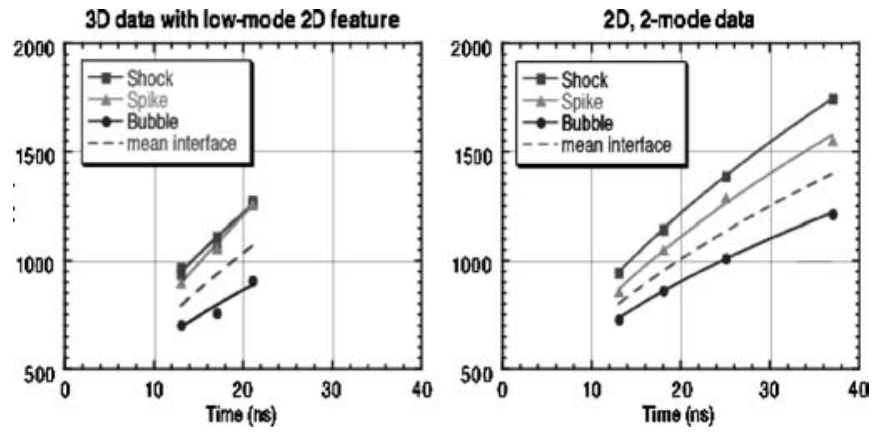

Figure 9. A comparison of the $3 \mathrm{D}$ perturbation experiment and 2D mode coupling perturbation experiment.

as a reference for the initial growth. At $17 \mathrm{~ns}$ the spikes are at the shock front and at $21 \mathrm{~ns}$ the spike structure is poorly resolved. The 2D and 3D data are compared in Figure 9. The plots show the position vs. time of the shock, spike and bubble. It is clear the spikes in the 3D perturbation have reached the shock while the spikes in the multi-mode 2D experiment are falling away from the shock front. This suggests the possibility that the superposition of a low-mode perturbation could significantly affect the extent of mixing in supernova.

To explore the increased spike penetration phenomenon a target with a recessed tracer perturbation was simulated in 2D with CALE. The target schematic used in this simulation can be seen in Figure 10a. The recessed tracer perturbation generates a jet-like flow enhancing the spike penetration. The CALE simulation at $17 \mathrm{~ns}$ can be 

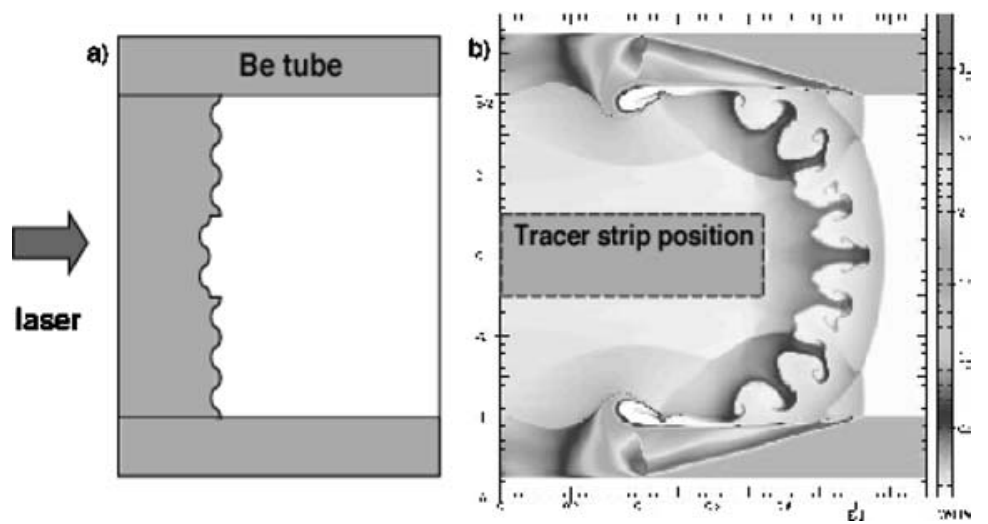

Figure 10. a) Target schematic with recessed tracer strip b) CALE simulation with recessed tracer strip.

seen in Figure 10b. From the diagnostic view, the spikes are essentially at the shock location. The enhanced spike penetration results primarily from a superposition of a jet-like flow as opposed to mode coupling.

\section{Conclusions}

Experiments have been performed on the Omega laser to study two possible mechanisms for enhanced radial transport in SN explosions. Significant improvements have been made in the target fabrication process, resulting in 3D surfaces of great precision. Future experiments in this series can now be more directly coupled to recent results from 3D stellar evolution models to address the issue of the effect of initial conditions on SN mixing.

\section{Acknowledgments}

This work is supported by the U.S. Department of Energy under grants DE-FG0399DP00284 and DE-FG03-00SF22021.

\section{References}

Boehly, T.R., Craxton, R.S. et al.: 1995, Rev. Sci. Intsr. 66(1), 508.

Fryxell, B., Muller, E. et al.: 1991, ApJ 367, 619.

Kifonidis, K., Plewa, T. et al.: 2000, ApJ Lett. 531(2), L123.

Meshkov, E.E.: 1969, Fluid Dyn. 4, 101.

Rayleigh, L.: 1900, Scientific Papers II. Cambridge, England.

Richtmyer, D.H.: 1960, Commun. Pure. Appl. Math 13, 297.

Ryutov, D.D., Drake, R.P. et al.: 1999, ApJ 518(2), 821.

Taylor, S.G.: 1950, Proc. R. Soc. A201, 192. 